\title{
Caracterização da Cobertura Florestal de Unidades de Conservação da Mata Atlântica
}

\author{
Roberto Silvério Neto ${ }^{1}$, Monique de Carvalho Bento ${ }^{1}$, \\ Sady Junior Martins da Costa de Menezes ${ }^{1}$, Fábio Souto Almeida ${ }^{1}$ \\ ${ }^{1}$ Departamento de Ciências do Meio Ambiente, Universidade Federal Rural \\ do Rio de Janeiro - UFRRJ, Três Rios/RJ, Brasil
}

\section{RESUMO}

O trabalho objetivou caracterizar a cobertura florestal de seis unidades de conservação da natureza no município de Três Rios, RJ: APA Bemposta; APA Lago do Caça e Pesca; APA Santa-Fé; APA Vale do Morro da Torre; MONA Encontro dos Três Rios; e o PNM Três Rios. Para localizar os fragmentos florestais foi utilizada a base de dados do satélite Landsat 5-TM, processada com os programas Erdas Imagine 9.2 e ArcGIS 10.0. Foram encontrados 527 fragmentos florestais, cobrindo uma área total de $7.075,77$ ha ( $26,88 \%$ da área das unidades de conservação). A APA Bemposta se destacou por possuir a maior porcentagem de área com cobertura florestal $(31,21 \%)$. O PNM Três Rios não apresentou fragmentos florestais com pelo menos 0,5 ha. As unidades de conservação do município apresentam um elevado número de fragmentos florestais relativamente pequenos, com formatos irregulares e grandemente isolados, o que contribui para a perda de biodiversidade.

Palavras-chave: Área de Proteção Ambiental, biodiversidade, fragmentos florestais, paisagem, sensoriamento remoto.

\section{Characterization of Forest Cover of Protected Areas of the Atlantic Forest}

\begin{abstract}
This work aimed to characterize the forest cover of protected areas in the municipality of Três Rios, Rio de Janeiro state, Brazil. Five Environmental Protection Areas (EPA) were studied: Bemposta, Lago do Caça e Pesca, Santa-Fé and Vale do Morro da Torre EPAs; Encontro dos Três Rios Natural Monument; and Três Rios Municipal Natural Park. The database of Landsat 5-TM satellite, processed with software Erdas Imagine 9.2 and ArcGIS 10.0, were used for the location of forest fragments. We found 527 forest fragments, covering a total area of 7075.77 ha $(26.88 \%$ of the protected areas). Bemposta Environmental Protection Area presents the highest percentage of forested area (31.21\%). Três Rios Municipal Natural Park presents no forest fragments with at least 0.5 ha. The protected areas show a large number of relatively small forest fragments with irregular shapes and greatly isolated, which contributes to loss of biodiversity.
\end{abstract}

Keywords: Environmental Protection Area, biodiversity, forest fragments, landscape, remote sensing. 


\section{INTRODUÇÃO}

A perda de biodiversidade tem preocupado autoridades públicas e a sociedade em geral, pois a extinção de espécies ocasiona a perda de serviços ecossistêmicos e gera prejuízos econômicos (Andrade \& Romeiro, 2009). No Brasil, o Bioma Mata Atlântica é uma área prioritária para a conservação da diversidade biológica, devido à sua elevada biodiversidade, elevado número de espécies endêmicas e avançado estado de degradação (Myers et al., 2000; Tabarelli et al., 2005). Dentre as fitofisionomias que ocorrem no Bioma Mata Atlântica estão as florestas estacionais semideciduais, que ocupavam originalmente uma área significativa do Bioma (IBGE, 1992) mas foram grandemente reduzidas (Ribeiro et al., 2011). As causas do desmatamento das florestas estacionais semideciduais incluem a implantação de pastagens, o cultivo de espécies agrícolas e o crescimento urbano (IBGE, 1992; Oliveira-Filho et al., 1994; Dean, 2002).

No século XIX, grande parte das florestas estacionais semideciduais do Vale do Paraíba foram desmatadas para a implantação da monocultura do café (Stein, 1985). Após a decadência da cultura do café, a pecuária tornou-se uma atividade importante na região, que também apresenta polos industriais (Soares, 2011). Assim, o histórico de ocupação do Vale do Paraíba gerou a perda de habitat e a fragmentação florestal (Devide et al., 2014). A região possui atualmente uma paisagem que é comumente observada na Mata Atlântica, pois apresenta uma reduzida cobertura florestal, dispersa em meio a pastagens, áreas agrícolas e urbanas (Ribeiro et al., 2011). Esse panorama contribui para a extinção de espécies e ameaça interações ecológicas, pondo em risco processos ecológicos importantes para os ecossistemas e para a economia (Viana \& Pinheiro, 1998; Vargas, 2011; Almeida et al., 2013).

Para proteger a diversidade biológica, a estratégia mais utilizada tem sido a conservação de ecossistemas naturais em unidades de conservação da natureza (Rylands \& Brandon, 2005; Almeida et al., 2011). No município de Três Rios, RJ, Médio Paraíba do Sul, foram estabelecidas seis unidades de conservação administradas pelo governo municipal, porém nessas áreas pouco restou da cobertura florestal original. Para a adequada gestão das áreas protegidas é fundamental a caracterização da sua cobertura florestal. Essa informação é essencial para o planejamento das ações de manejo necessárias para a gestão da biodiversidade.

Desse modo, o presente trabalho objetiva caracterizar a cobertura florestal das unidades de conservação do município de Três Rios, analisando o tamanho, a forma e o nível de isolamento dos fragmentos florestais presentes nas áreas protegidas.

\section{MATERIAL E MÉTODOS}

O presente trabalho foi conduzido no município de Três Rios, estado do Rio de Janeiro (Figura 1), que apresenta $326,136 \mathrm{~km}^{2}$ de área total e 77.432 habitantes (IBGE, 2013). No município predominam os argissolos e o clima é o mesotérmico, sendo a temperatura mínima de $14,4^{\circ} \mathrm{C}$, a temperatura máxima de $37,4^{\circ} \mathrm{C}$ e a precipitação de $1.300 \mathrm{~mm}$ por ano (Nascimento \& Machado, 2009; Gomes et al., 2013). O município possui seis unidades de conservação da natureza, todas municipais: Área de Proteção Ambiental Bemposta (APA Bemposta), com 19.942,49 ha; Área de Proteção Ambiental Lago do Caça e Pesca (APA Lago do Caça e Pesca), que apresenta 32,94 ha; Área de Proteção Ambiental Santa-Fé (APA Santa-Fé), com 1.841,22 ha; Área de Proteção Ambiental Vale do Morro da Torre (APA Vale do Morro da Torre), que possui 4.236,58 ha; Monumento Natural Municipal Encontro dos Três Rios (MONA Encontro dos Três Rios), com área total de 267,53 ha; e o Parque Natural Municipal de Três Rios (PNM Três Rios), que apresenta 26,2 ha.

Para localizar os fragmentos florestais nas unidades de conservação de Três Rios foi utilizada a base de dados do satélite orbital Landsat 5-TM, ponto/órbita 217/075 do dia 13 de agosto de 2011, fornecida de forma gratuita pelo Instituto Nacional de Pesquisas Espaciais (INPE) no seu site institucional. Foi utilizado o programa Erdas Imagine 9.2 para efetuar a junção das sete bandas espectrais obtidas do sensor TM (Thematic Mapper), do satélite Landsat 5. Utilizou-se a configuração da imagem raster obtida do sensor TM em 5R, 4G, 3B para a visualização dos fragmentos, pois essa configuração caracterizou-se pela melhor detecção dos alvos, evidenciando, portanto, a vegetação. Em seguida, dentro da imagem raster obtida foi enquadrado o polígono referente ao município de Três Rios por meio do arquivo vetorial em formato shapefile, disponibilizado gratuitamente pelo Instituto Nacional 


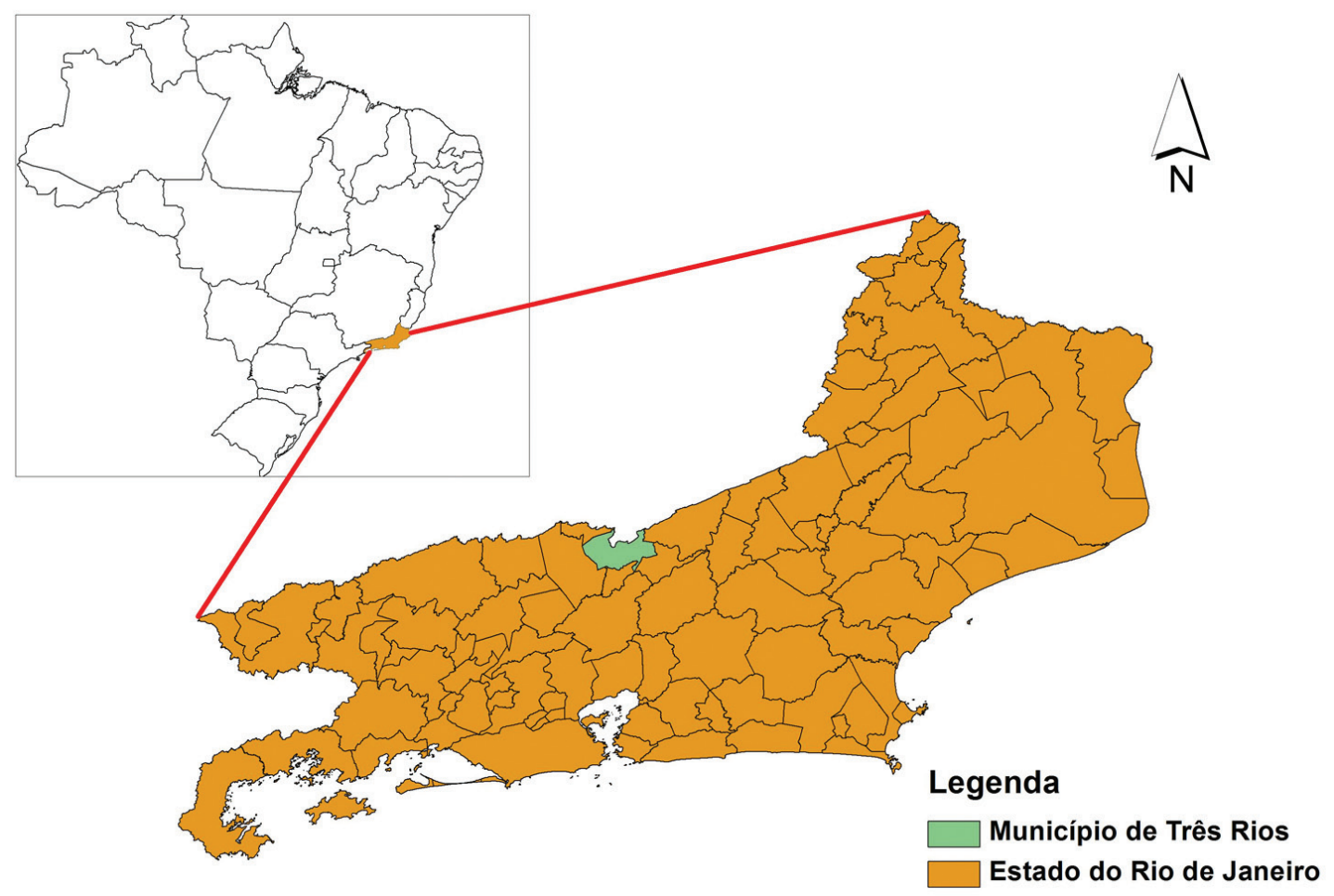

Figura 1. Localização do município de Três Rios, estado do Rio de Janeiro.

Figure 1. Location of the city of Três Rios, Rio de Janeiro State.

de Geografia e Estatística (IBGE), para recortar a sua área, com o objetivo de facilitar o processamento dos dados no programa, sendo processada apenas a área correspondente ao trabalho em questão. O próximo passo foi realizar a classificação supervisionada, com o intuito de identificar os fragmentos florestais presentes no município.

Com o objetivo de estudar apenas os fragmentos significativos para o manejo da paisagem, visando a gestão da biodiversidade, e evitar que uma grande árvore isolada ou um pequeno agrupamento de árvores fossem caracterizados como florestas, foram analisadas somente as áreas classificadas como fragmentos florestais de pelo menos 0,5 ha. Utilizou-se o programa ArcGIS 10.0 para efetuar a qualificação e quantificação dos fragmentos florestais. Utilizamos um conjunto de ortofotos do município de Três Rios obtidas no IBGE para o ano de 2005 (ano do voo e obtenção dessas ortofotos), efetuando-se também saídas a campo para validar a fotointerpretação e os resultados obtidos pela classificação supervisionada.

A delimitação das unidades de conservação de Três Rios foi realizada por meio de seus memoriais descritivos, projetando-as nas imagens trabalhadas nos programas citados. Foi realizado um recorte da imagem das áreas das unidades de conservação de Três Rios e obteve-se a área, o perímetro e o nível de isolamento de cada fragmento florestal presente nas unidades de conservação por meio de ferramentas (comandos específicos) no ArcGIS 10.0.

Para estudar o nível de isolamento foi medida a distância de um fragmento florestal para o seu vizinho mais próximo. $\mathrm{O}$ índice de circularidade foi calculado para cada fragmento florestal (Borges et al., 2004; Amaral et al., 2009) conforme as Expressões 1, 2 e 3:

IC $=\sqrt{ }\left(A_{1} / A_{2}\right)$

$\mathrm{A}_{2}=\pi \mathrm{R}^{2}$

$\mathrm{R}=\mathrm{P} / 2 \pi$

em que: $\mathrm{IC}=$ índice de circularidade; $\mathrm{A}_{1}=$ área do fragmento florestal; $\mathrm{A}_{2}=$ área de um círculo com perímetro igual ao do fragmento florestal; $\mathrm{R}=$ raio do círculo; e $\mathrm{P}$ = perímetro.

Quanto mais próximo o índice estiver de 1, mais circular será o remanescente florestal. Por outro 
lado, quanto mais alongada ou irregular for a área do fragmento florestal, mais próximo de 0 será o índice (Borges et al., 2004; Amaral et al., 2009).

Com base nesses dados foram obtidas a área com cobertura florestal e a porcentagem da área total da unidade de conservação coberta por florestas. Além disso, os fragmentos florestais foram agrupados em classes de tamanho, de índice de circularidade e de nível de isolamento.

\section{RESULTADOS E DISCUSSÃO}

Foram encontrados 527 fragmentos florestais nas unidades de conservação, cobrindo uma área de $7.075,77$ ha. No total, a porcentagem da área das unidades de conservação coberta por florestas foi de $26,88 \%$.

O Parque Natural Municipal de Três Rios não apresentou fragmentos florestais com pelo menos 0,5 ha. Essa unidade de conservação possui apenas 26,2 ha e a maior parte da sua área é ocupada pelo Rio Paraíba do Sul. A APA Bemposta se destacou como a que possui maior porcentagem da área com cobertura florestal (Tabela 1). Essa unidade de conservação é a maior do município (Figura 2), seguida da APA Vale do Morro da Torre (Figura 3). As demais unidades de conservação apresentaram uma porcentagem de cobertura florestal expressivamente menor que a da APA Bemposta, variando de 9,68\% (APA Lago do Caça e Pesca, Figura 4) até 13,61\% (APA Santa-Fé, Figura 5), sendo semelhante à proporção de cobertura florestal observada para a Mata Atlântica em geral, aproximadamente 12\% (Ribeiro et al., 2011).

A perda de habitat é apontada como uma das mais importantes ameaças à biodiversidade no mundo (Primack \& Rodrigues, 2001). Em geral, populações bióticas presentes em paisagens com baixa porcentagem de cobertura florestal são propensas à perda de diversidade genética e estão sujeitas a escassez de recursos, como alimento e abrigo (Hanski, 2011). Com isso, o número de indivíduos dessas populações tende a diminuir, chegando à extinção local. Espécies generalistas podem sobreviver em paisagens com uma pequena proporção de cobertura florestal por utilizarem recursos presentes nos arredores das florestas, mas a diversidade de espécies como um todo tende a ser menor nessas paisagens (Andrén, 1994).

A APA Bemposta é a unidade de conservação que possui mais fragmentos florestais, seguida da APA Morro da Torre (Tabela 2). É importante observar que o número de fragmentos florestais da APA Bemposta é mais de quatro vezes maior que o da APA Morro da Torre. Por outro lado, a APA Lago do Caça e Pesca possui apenas um fragmento florestal.

A maioria dos fragmentos florestais encontrados nas unidades de conservação de Três Rios possui menos de um hectare. O número de fragmentos entre um e cinco hectares também é expressivo. Todavia, os fragmentos com menos de cinco hectares contribuem relativamente pouco para a área total de cobertura florestal. Na APA Bemposta, apenas 17 fragmentos florestais com área maior que 100 ha representam $70,67 \%$ da área com cobertura florestal na unidade de conservação. $\mathrm{Na}$ APA do Morro da Torre e na APA Santa-Fé, os poucos fragmentos com área acima de 20 ha também foram os que contribuíram mais para a cobertura florestal total. Já no MONA Encontro dos Três Rios não foram observados fragmentos florestais com áreas maiores que 20 ha. O único fragmento florestal encontrado na APA Lago do Caça e Pesca possui 3,19 ha. A maioria dos fragmentos florestais da Mata Atlântica possui pequeno tamanho e os fragmentos grandes representam a maior parte da área com cobertura florestal (Borges et al., 2004; Amaral et al., 2009; Greggio et al., 2009).

Tabela 1. Área total, área com cobertura florestal e porcentagem da área total com cobertura florestal nas unidades de conservação do município de Três Rios, RJ.

Table 1. Total area, area of forest cover and the percentage of the total area with forest cover in protected areas in the city of Três Rios, Rio de Janeiro State.

\begin{tabular}{lccrrc}
\multicolumn{1}{c}{ Atributos } & $\begin{array}{c}\text { APA Bem- } \\
\text { posta }\end{array}$ & $\begin{array}{c}\text { APA Lago do } \\
\text { Caça e Pesca }\end{array}$ & $\begin{array}{c}\text { APA Morro } \\
\text { da Torre }\end{array}$ & $\begin{array}{c}\text { MONA } \\
\text { APA Santa-Fé }\end{array}$ & $\begin{array}{c}\text { Encontro dos } \\
\text { Três Rios }\end{array}$ \\
\hline Área total (ha) & $19.942,49$ & 32,94 & $4.236,58$ & $1.841,22$ & 267,53 \\
Área com cobertura florestal (ha) & $6.224,25$ & 3,19 & 567,86 & 250,59 & 29,88 \\
Cobertura florestal (\%) & 31,21 & 9,68 & 13,40 & 13,61 & 11,17 \\
\hline
\end{tabular}



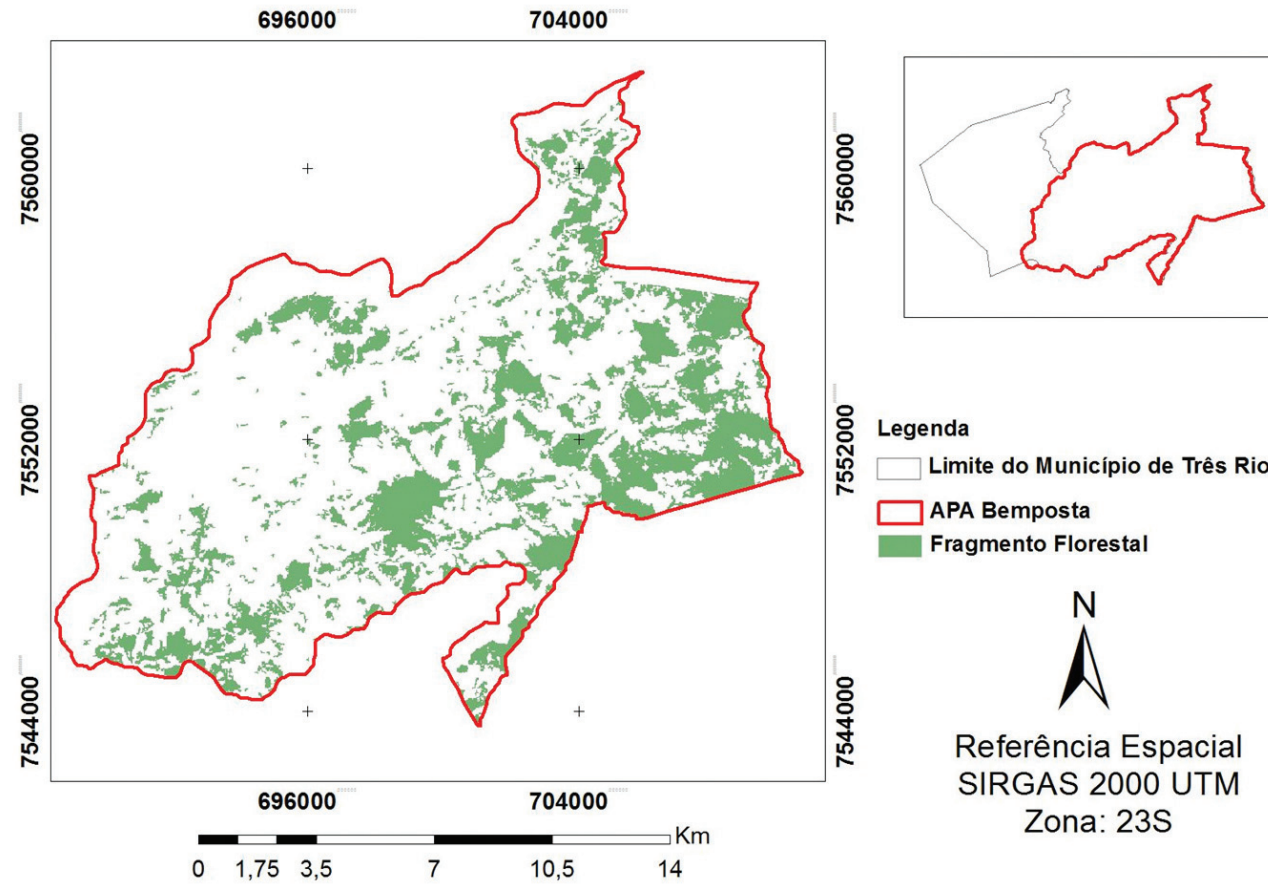

\section{응 Legenda \\ 莡 $\square$ Limite do Município de Três Rios

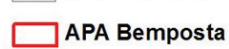 \\ Fragmento Florestal}

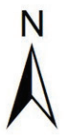

Referência Espacial SIRGAS 2000 UTM Zona: 23S

Figura 2. Localização dos fragmentos florestais na APA Bemposta, município de Três Rios, RJ.

Figure 2. Location of forest fragments in the Bemposta Environmental Protection Area, city of Três Rios, Rio de Janeiro State.
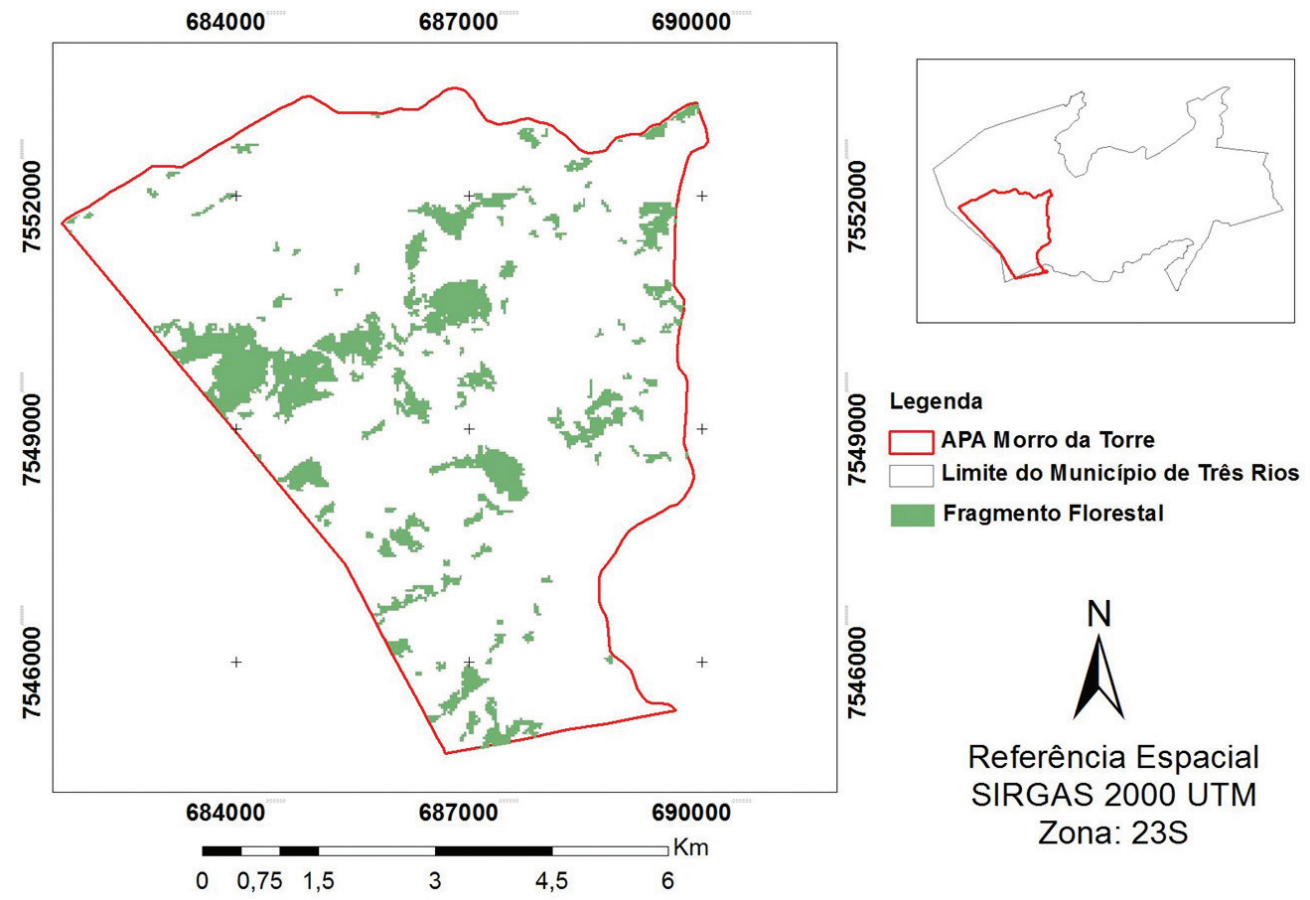

\section{Referência Espacial SIRGAS 2000 UTM Zona: $23 S$}

Figura 3. Localização dos fragmentos florestais na APA Vale do Morro da Torre, município de Três Rios, RJ.

Figure 3. Location of forest fragments in the Vale do Morro da Torre Environmental Protection Area, city of Três Rios, Rio de Janeiro State. 

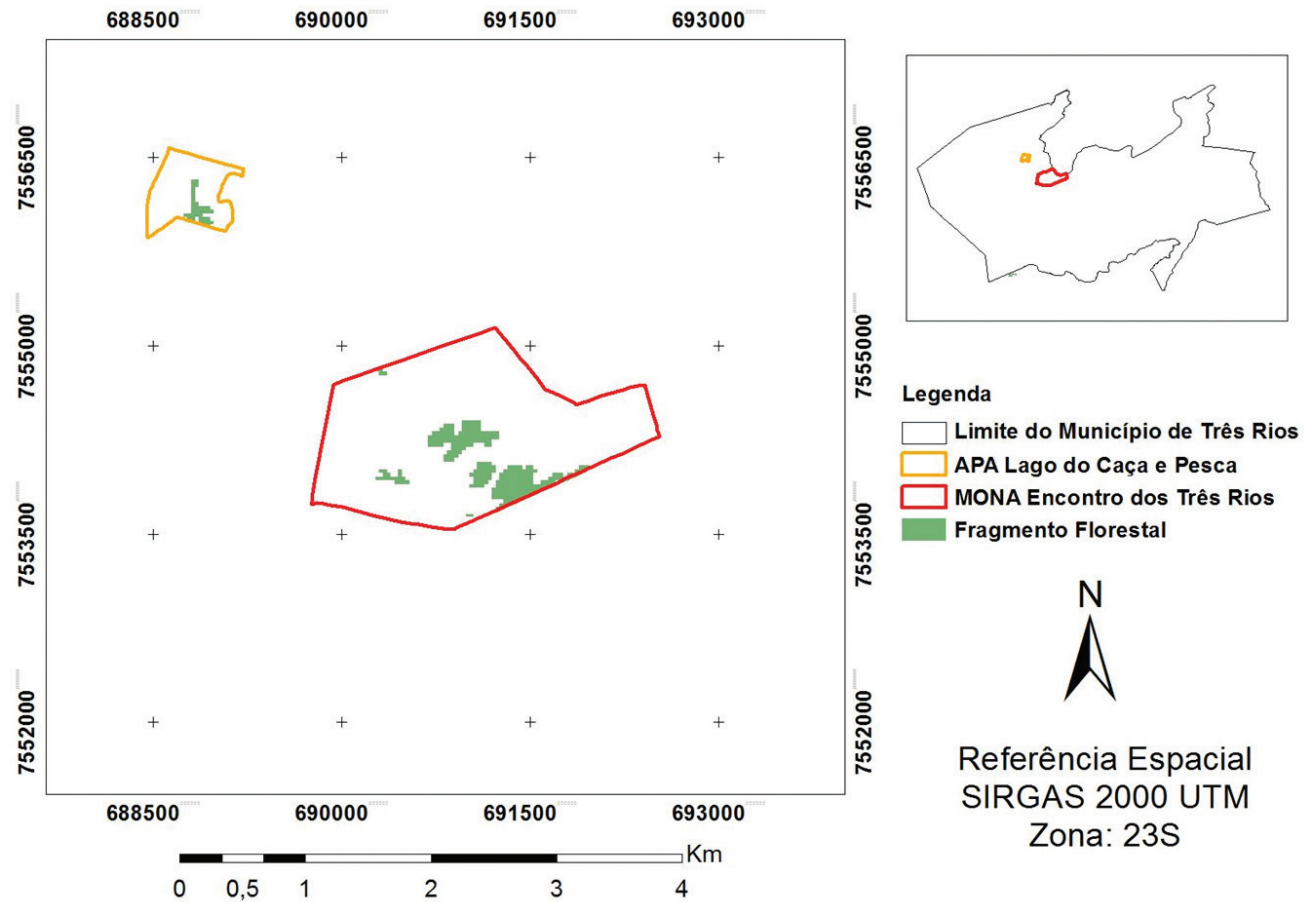

\section{Referência Espacial SIRGAS 2000 UTM Zona: $23 S$}

Figura 4. Localização dos fragmentos florestais na APA Lago do Caça e Pesca e no MONA Encontro dos Três Rios, município de Três Rios, RJ.

Figure 4. Location of forest fragments in the Lago do Caça e Pesca Environmental Protection Area and in the Encontro dos Três Rios Natural Monument, in the city of Três Rios, Rio de Janeiro State.
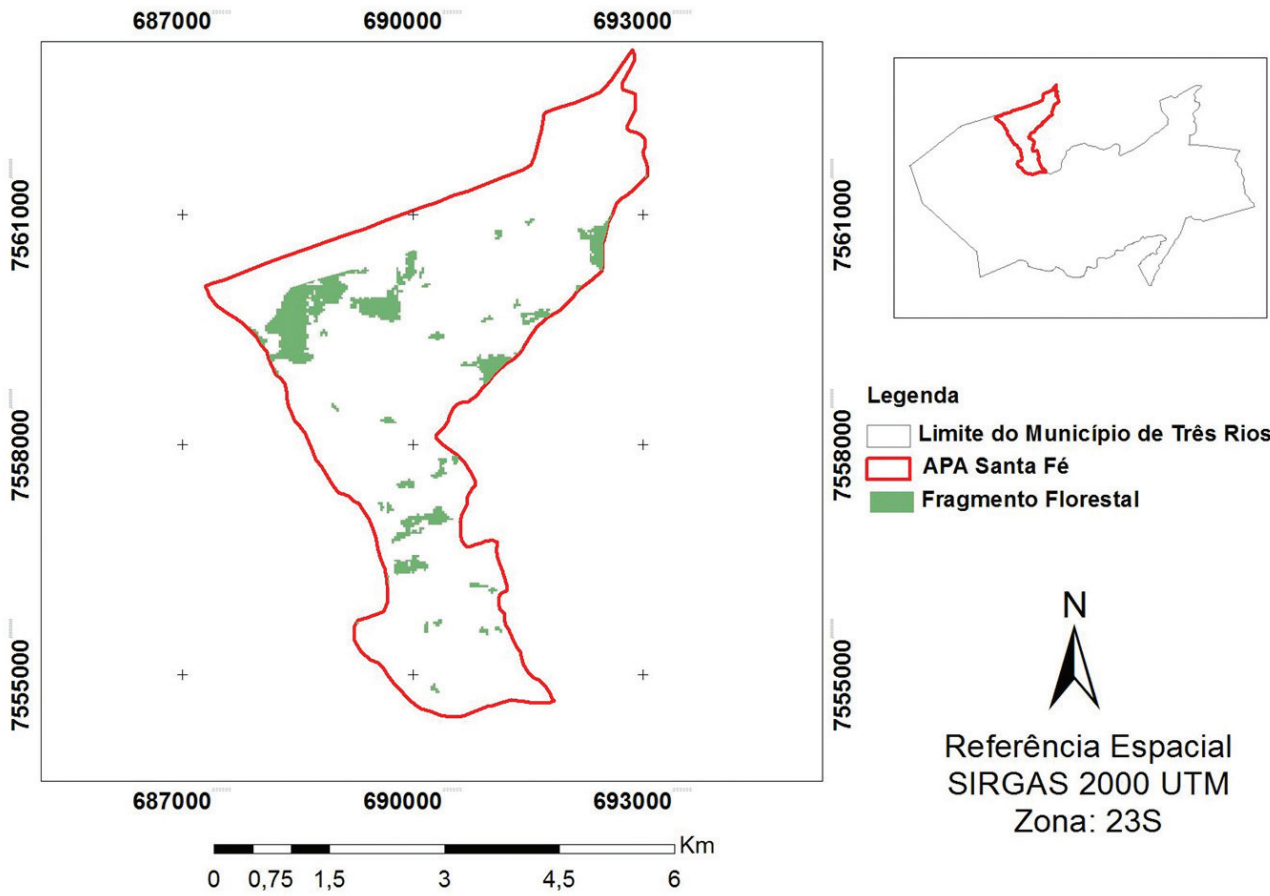

Referência Espacial SIRGAS 2000 UTM Zona: $23 S$

Figura 5. Localização dos fragmentos florestais na APA Santa-Fé, município de Três Rios, RJ.

Figure 5. Location of forest fragments in the Santa-Fé Environmental Protection Area, city of Três Rios, Rio de Janeiro State. 
Tabela 2. Número de fragmentos florestais $(\mathrm{N})$, área e a porcentagem da área total com cobertura florestal (\%) por classe de tamanho, nas unidades de conservação do município de Três Rios, RJ.

Table 2. Number of forest fragments $(\mathrm{N})$, area and the percentage of forest cover by size class, in protected areas of the city of Três Rios, Rio de Janeiro State.

\begin{tabular}{|c|c|c|c|c|c|c|c|c|c|c|c|c|}
\hline \multirow{2}{*}{$\begin{array}{l}\text { Classes de } \\
\text { área (ha) }\end{array}$} & \multicolumn{3}{|c|}{ APA Bemposta } & \multicolumn{3}{|c|}{ APA Morro da Torre } & \multicolumn{3}{|c|}{ APA Santa Fé } & \multicolumn{3}{|c|}{$\begin{array}{c}\text { MONA Encontro dos } \\
\text { Três Rios } \\
\end{array}$} \\
\hline & $\mathbf{N}$ & Área & $\%$ & $\mathbf{N}$ & Área & $\%$ & $\mathbf{N}$ & Área & $\%$ & $\mathbf{N}$ & Área & $\%$ \\
\hline $0,5-11,0$ & 158 & 111,69 & 1,79 & 49 & 33,84 & 5,96 & 18 & 12,00 & 4,79 & 2 & 1,44 & 4,82 \\
\hline $1,0-15,0$ & 153 & 349,03 & 5,61 & 30 & 69,48 & 12,24 & 6 & 13,32 & 5,32 & 2 & 4,68 & 15,66 \\
\hline $5,0-110,0$ & 24 & 170,09 & 2,73 & 4 & 32,94 & 5,80 & 2 & 13,25 & 5,29 & 1 & 9,18 & 30,72 \\
\hline $10,0-120,0$ & 17 & 234,57 & 3,77 & 6 & 89,73 & 15,80 & 2 & 29,70 & 11,85 & 1 & 14,58 & 48,80 \\
\hline $20,0-150,0$ & 19 & 626,76 & 10,07 & 5 & 137,48 & 24,21 & 2 & 74,47 & 29,72 & 0 & 0,00 & 0,00 \\
\hline $50,0-1100,0$ & 5 & 333,36 & 5,36 & 1 & 50,40 & 8,88 & 0 & 0,00 & 0,00 & 0 & 0,00 & 0,00 \\
\hline$>100,0$ & 17 & $4.398,75$ & 70,67 & 1 & 153,99 & 27,12 & 1 & 107,85 & 43,04 & 0 & 0,00 & 0,00 \\
\hline Total & 393 & $6.224,25$ & 100,00 & 96 & 567,86 & 100,00 & 31 & 250,59 & 100,00 & 6 & 29,88 & 100,00 \\
\hline
\end{tabular}

A existência de fragmentos florestais de tamanho elevado é fundamental para a conservação da biodiversidade, pois podem suportar populações com um grande número de indivíduos e apresentam maior diversidade de habitats (Almeida et al., 2011). Assim, geralmente possuem maior biodiversidade que os remanescentes florestais pequenos (Viana \& Pinheiro, 1998). Os fragmentos florestais de pequeno tamanho geralmente não podem manter grandes populações, especialmente de animais de grande porte. Populações com um grande número de indivíduos têm maior probabilidade de persistir por longo período que as populações pequenas, que possuem menor diversidade genética, menor plasticidade evolucionária, estão mais sujeitas à endogamia, à variação demográfica e às catástrofes ambientais (Primack \& Rodrigues, 2001).

Quanto aos índices de circularidade, os valores médios foram semelhantes para todas as unidades de conservação (Tabela 3). Na APA Bemposta, na APA Morro da Torre e na APA Santa-Fé, a classe de índice de circularidade entre 0,6 e 0,8 foi a que apresentou o maior número de fragmentos florestais. Porém, mais da metade dos fragmentos florestais na APA Bemposta e na APA Morro da Torre possuem índice abaixo de 0,6 . Além disso, um pequeno número de fragmentos florestais teve índice de circularidade acima de 0,8 . Já no MONA Encontro dos Três Rios os seis fragmentos existentes se dividiram igualmente entre as classes centrais. Assim, os dados demostram que um número expressivo de remanescentes florestais das unidades de conservação de Três Rios apresenta forma alongada ou irregular, o que pode comprometer a conservação da biodiversidade na região (Saunders et al., 1991; Gimenes \& Anjos, 2003; Laurance \& Vasconcelos, 2009).

O nível de isolamento médio dos fragmentos florestais é menor na APA Bemposta, seguida da APA Morro da Torre (Tabela 4). Por outro lado, o nível de isolamento dos fragmentos florestais na APA Santa-Fé e, principalmente, no MONA Encontro dos Três Rios é especialmente preocupante. O isolamento dos fragmentos florestais isola populações de animais e plantas, diminuindo o fluxo gênico e aumentando a endogamia (Seoane et al., 2000; Laurance \& Vasconcelos, 2009). Isso pode ocasionar perda de diversidade genética e de flexibilidade evolucionária, aumento da ocorrência de doenças genéticas e, consequentemente, aumento da probabilidade de extinção local (Primack \& Rodrigues, 2001).

A APA Bemposta é a que apresenta a paisagem mais adequada para a conservação da biodiversidade. Todavia, mesmo essa unidade de conservação apresenta inúmeros remanescentes florestais pequenos, com elevado nível de isolamento e com formas alongadas ou irregulares. Assim, é necessário que os responsáveis pela gestão das unidades de conservação realizem intervenções por meio de programas de manejo, com $\mathrm{o}$ intuito de proteger os remanescentes florestais e melhorar a condição ambiental dessas áreas, visando a conservação da diversidade biológica.

É necessário aumentar a cobertura florestal das unidades de conservação, ligar os remanescentes florestais e diminuir o efeito de borda. Nesse sentido, podem ser implementados corredores ecológicos 
Tabela 3. Número de fragmentos florestais $(\mathrm{N})$ por classe de índice de circularidade, para as unidades de conservação no município de Três Rios, RJ.

Table 3. Number of forest fragments by circularity index class, in protected areas of city of Três Rios, Rio de Janeiro State.

\begin{tabular}{|c|c|c|c|c|c|c|c|c|}
\hline \multirow{2}{*}{$\begin{array}{l}\text { Classes de índice } \\
\text { de circularidade }\end{array}$} & \multicolumn{2}{|c|}{ APA Bemposta } & \multicolumn{2}{|c|}{ APA Morro da Torre } & \multicolumn{2}{|c|}{ APA Santa-Fé } & \multicolumn{2}{|c|}{$\begin{array}{c}\text { MONA Encontro } \\
\text { dos Três Rios }\end{array}$} \\
\hline & $\mathbf{N}$ & $\%$ & $\mathbf{N}$ & $\%$ & $\mathbf{N}$ & $\%$ & $\mathbf{N}$ & $\%$ \\
\hline $0,0-10,4$ & 69 & 17,60 & 16 & 16,67 & 4 & 12,90 & 0 & 0,00 \\
\hline $0,4-10,6$ & 151 & 38,52 & 34 & 35,42 & 7 & 22,58 & 3 & 50,00 \\
\hline $0,6-10,8$ & 164 & 41,84 & 43 & 44,79 & 20 & 64,52 & 3 & 50,00 \\
\hline$>0,8$ & 8 & 2,04 & 3 & 3,13 & 0 & 0,00 & 0 & 0,00 \\
\hline Índice médio $\pm \mathrm{EP}$ & \multicolumn{2}{|c|}{$0,55 \pm 0,01$} & \multicolumn{2}{|c|}{$0,57 \pm 0,01$} & \multicolumn{2}{|c|}{$0,59 \pm 0,02$} & \multicolumn{2}{|c|}{$0,57 \pm 0,05$} \\
\hline
\end{tabular}

Tabela 4. Classes de nível de isolamento dos fragmentos florestais das unidades de conservação no município de Três Rios, RJ.

Table 4. Classes of isolation level of forest fragments in protected areas, city of Três Rios, Rio de Janeiro State.

\begin{tabular}{|c|c|c|c|c|c|c|c|c|}
\hline \multirow{2}{*}{$\begin{array}{l}\text { Classes de nível de } \\
\text { isolamento (m) }\end{array}$} & \multicolumn{2}{|c|}{ APA Bemposta } & \multicolumn{2}{|c|}{$\begin{array}{c}\text { APA Morro da } \\
\text { Torre } \\
\end{array}$} & \multicolumn{2}{|c|}{ APA Santa-Fé } & \multicolumn{2}{|c|}{$\begin{array}{c}\text { MONA Encontro } \\
\text { dos Três Rios }\end{array}$} \\
\hline & $\mathbf{N}$ & $\%$ & $\mathbf{N}$ & $\%$ & $\mathbf{N}$ & $\%$ & $\mathbf{N}$ & $\%$ \\
\hline Até 30,0 & 87 & 22,14 & 18 & 18,75 & 5 & 16,13 & 0 & 0,00 \\
\hline $30,0-150,0$ & 44 & 11,20 & 6 & 6,25 & 3 & 9,68 & 1 & 16,67 \\
\hline $50,0-1100,0$ & 140 & 35,62 & 29 & 30,21 & 9 & 29,03 & 0 & 0,00 \\
\hline $100,0-1200,0$ & 74 & 18,83 & 28 & 29,17 & 3 & 9,68 & 2 & 33,33 \\
\hline $200,0-1300,0$ & 27 & 6,87 & 12 & 12,50 & 1 & 3,23 & 0 & 0,00 \\
\hline$>300,0$ & 21 & 5,34 & 3 & 3,13 & 10 & 32,26 & 3 & 50,00 \\
\hline Média \pm EP $(\mathrm{m})$ & \multicolumn{2}{|c|}{$103,03 \pm 5,10$} & \multicolumn{2}{|c|}{$122,09 \pm 14,65$} & \multicolumn{2}{|c|}{$209,87 \pm 36,87$} & \multicolumn{2}{|c|}{$742,83 \pm 493,70$} \\
\hline
\end{tabular}

e reflorestamentos que visem tornar os fragmentos florestais mais circulares (Amador, 2003; Seoane et al., 2010). Corredores ecológicos ligando os fragmentos florestais podem colaborar para o aumento do fluxo gênico entre as populações e permitir a manutenção de um maior número de espécies nas unidades de conservação. Os reflorestamentos realizados com o intuito de tornar os fragmentos florestais mais circulares diminuem a porcentagem de área sob o efeito de borda. Essas medidas podem contribuir para aumentar a cobertura florestal das unidades de conservação e para a conservação da biodiversidade.

Os custos para a realização de reflorestamentos podem dificultar a sua execução. Todavia cabe ressaltar que a restauração florestal nas unidades de conservação de Três Rios pode ser realizada com recursos do ICMS Verde, pois estima-se que o município irá receber R\$ 1.711.052 em 2014, sendo 91,7\% desse valor devido às unidades de conservação presentes no município (SEA, 2014). Além disso, técnicas alternativas ao plantio adensado de espécies arbóreas podem ser utilizadas para diminuir os custos da restauração florestal, como as técnicas de nucleação, que podem ser 34\% menos custosas que a convencional (Bechara, 2006). Os sistemas agroflorestais também podem ser utilizados para a restauração florestal, por reduzirem os custos de implantação e favorecerem o crescimento das árvores (Almeida et al., 2011; Daronco et al., 2012), viabilizando a conectividade entre remanescentes florestais (Sollberg et al., 2014).

As unidades de conservação são susceptíveis à pressão antrópica e às variações ambientais, que podem levar à perda de espécies, especialmente em paisagens com habitats fragmentados (Laurance et al., 2012), como as paisagens observadas em Três Rios. As unidades de conservação de Três Rios estão particularmente sujeitas às ações antrópicas, pois as Áreas de Proteção Ambiental e a MONA Encontro dos Três Rios apresentam ocupação humana. Além disso, o PNM Três Rios tem tamanho reduzido e a visitação pública é permitida. 
Assim, os programas de manejo dessas unidades de conservação devem contemplar medidas educativas e a fiscalização. Tais medidas podem minimizar diversas ameaças à biodiversidade, como o desmatamento ilegal e as queimadas (Tebaldi et al., 2013), que são frequentes nas pastagens da região.

\section{CONCLUSÃO}

O estado atual da paisagem das unidades de conservação de Três Rios contribui para a perda de biodiversidade. Assim, programas de manejo devem ser implementados para reverter esse quadro, englobando a criação de corredores ecológicos e de reflorestamentos que visem aumentar o tamanho dos remanescentes florestais.

A APA Bemposta é a unidade de conservação que apresenta a paisagem mais propícia para a conservação da biodiversidade. Todavia, mesmo essa unidade de conservação necessita de ações para maximizar a conservação de espécies na área.

\section{AGRADECIMENTOS}

À PROPPG/UFRRJ pela bolsa de iniciação científica (PROIC) concedida a RSN. Aos revisores anônimos pelas sugestões que muito contribuíram para a melhoria do artigo.

\section{STATUS DA SUBMISSÃO}

Recebido: 29 ago., 2013

Aceito: 10 set., 2014

\section{AUTOR(ES) PARA CORRESPONDÊNCIA}

\section{Fábio Souto Almeida}

Departamento de Ciências do Meio Ambiente, Universidade Federal Rural do Rio de Janeiro UFRRJ, CEP 25802-100, Três Rios, RJ, Brasil e-mail: fbio_almeida@yahoo.com.br

\section{REFERENCIAS}

Almeida FS, Gomes DS, Queiroz JM. Estratégicas para a conservação da biodiversidade biológica em florestas fragmentadas. Ambiência 2011; 7(2): 367-382.
Almeida FS, Mayhé-Nunes AJ, Queiroz JM. The importance of poneromorph ants for seed dispersal in altered environments. Sociobiology 2013; 60(3): 229-235. http://dx.doi.org/10.13102/sociobiology.v60i3.229-235.

Amador DB. Restauração de ecossistemas com sistemas agroflorestais. In: Kageyama PY, Oliveira RE, Moraes, LFD, Engel, VL, Gandara FB, organizadores. Restauração ecológica de ecossistemas naturais. Botucatu: FEPAF; 2003.

Amaral MVF, Souza AL, Soares VP, Soares CPB, Martins SV, Leite HG et al. Dinâmica da cobertura vegetal (1980-2004) em um projeto de produção florestal, nos municípios de Bugre e Ipaba, MG. Revista Árvore 2009; 33(2): 315-325. http://dx.doi.org/10.1590/S0100-67622009000200013.

Andrade DC, Romeiro AR. Serviços ecossistêmicos e sua importância para o sistema econômico e bem-estar humano. Texto para Discussão, IE/UNICAMP 2009; 155: 1-44.

Andrén H. Effects of habitat fragmentation on birds and mammals in landscapes with different proportions of suitable habitat: a review. Oikos 1994; 71(3): 355-366. http://dx.doi.org/10.2307/3545823.

Bechara FC. Unidades demonstrativas de restauração ecológica através de técnicas nucleadoras: Floresta Estacional Semidecidual, cerrado e restinga [tese]. Piracicaba: Escola Superior de Agricultura Luiz de Queiroz, Universidade de São Paulo; 2006.

Borges LFR, Scolforo JR, Oliveira AD, Mello JM, Acerbi FW Jr, Freitas GD. Inventário de fragmentos florestais nativos e propostas para seu manejo e o da paisagem. Cerne 2004; 10(1): 22-38.

Daronco C, Melo ACG, Machado JAR. Consórcio de espécies nativas da floresta estacional semidecidual com mandioca (Manihot suculenta Crant) para restauração de mata ciliar. Revista Árvore 2012; 36(2): 291-299. http:// dx.doi.org/10.1590/S0100-67622012000200010.

Dean W. A Ferro e Fogo: A História e a Devastação da Mata Atlântica Brasileira. São Paulo: Cia das Letras; 2002.

Devide ACP, Castro CM, Ribeiro RLD, Abboud ACS, Pereira MG, Rumjanek NG. História ambiental do Vale do Paraíba Paulista, Brasil. Revista Biociências 2014; 20(1): 12-29.

Gimenes MR, Anjos L. Efeitos da fragmentação florestal sobre as comunidades de aves. Acta Scientiarum. Biological Sciences 2003; 25(2): 391-402.

Gomes OVO, Marques ED, Souza MDC, Silva-Filho EV. Influência antrópica nas águas superficiais da cidade de Três Rios, RJ. Geochimica Brasiliensis 2013; 27(1): 77-86. http://dx.doi.org/10.5327/Z0102-9800201300010007.

Greggio TC, Pissarra TCT, Rodrigues FM. Avaliação dos fragmentos florestais do município de JaboticabalSP. Revista Árvore 2009; 33(1): 117-124. http://dx.doi. org/10.1590/S0100-67622009000100012.

Hanski I. Habitat loss, the dynamics of biodiversity, and a perspective on conservation. Ambio 2011; 40(3): 
248-255. http://dx.doi.org/10.1007/s13280-011-0147-3. PMid:21644453

Instituto Brasileiro de Geografia e Estatística - IBGE. Censo demográfico 2010. 2013. [citado 2013 ago. 17]. Disponível em: http://www.ibge.gov.br/cidadesat/xtras/perfil.php? codmun $=330600 \&$ search $=$ rio-de-janeiro|tres-rios.

Instituto Brasileiro de Geografia e Estatística - IBGE. Manual técnico da vegetação brasileira. Rio de Janeiro: IBGE; 1992.

Laurance WF, Useche DC, Rendeiro J, Kalka M, Bradshaw CJA, Sloan SP et al. Averting biodiversity collapse in tropical forest protected areas. Nature 2012; 489(7415): 290-294. http://dx.doi.org/10.1038/nature11318. PMid:22832582

Laurance WF, Vasconcelos HL. Consequências ecológicas da fragmentação florestal na Amazônia. Oecologia Brasiliensis 2009; 13(3): 434-451. http://dx.doi.org/10.4257/ oeco.2009.1303.03.

Myers N, Mittermeier RA, Mittermeier CG, da Fonseca GA, Kent J. Biodiversity hotspots for conservation priorities. Nature 2000; 403(6772): 853-858. http://dx.doi. org/10.1038/35002501. PMid:10706275

Nascimento ELM, Machado PS. Café, impacto ambiental e paisagem: uma abordagem interdisciplinar. 2009. [citado 2009 jun. 06]. Disponível em: http://www.prefeitura. alemparaiba.org/pdf/2013/arquivos/Projeto/3Cafe.pdf.

Oliveira-Filho AT, Vilela EA, Gavilaness ML, Carvalho DA. Comparison of the woody flora and soils of six areas of montane semideciduous forest in southern Minas Gerais, Brazil. Edinburgh Journal of Botany 1994; 51(3): 355-389. http://dx.doi.org/10.1017/S0960428600001839.

Primack RB, Rodrigues E. Biologia da conservação. Londrina: Editora Rodrigues; 2001.

Ribeiro MC, Martensen AC, Metzger JP, Tabarelli M, Scarano F, Fortin MJ. The Brazilian Atlantic Forest: a shrinking biodiversity hotspot. In: Zachos FE, Habel JC, editores. Biodiversity hotspots: distribution and protection of conservation priority areas. Heidelberg: Springer; 2011. http://dx.doi.org/10.1007/978-3-642-20992-5_21.

Rylands AB, Brandon K. Unidades de conservação brasileiras. Megadiversidade 2005; 1: 27-35.

Saunders DA, Hobbs RJ, Margules CR. Biological consequences of ecosystem fragmentation: a review.
Conservation Biology 1991; 5(1): 18-32. http://dx.doi. org/10.1111/j.1523-1739.1991.tb00384.x.

Secretaria de Estado do Ambiente - SEA. ICMS Verde. 2014. [citado 2014 ago. 15]. Disponível em: http:// download. rj.gov.br/documentos/10112/1878866/DLFE-70133.pdf/ estimative distribuicao2014.pdf.

Seoane CES, Kageyama PY, Sebbenn AM. Efeitos da fragmentação florestal na estrutura genética de populações de Esenbeckia leiocarpa Engl. (Guarantã). Scientia Forestalis 2000; 57: 123-139.

Seoane CESS, Diaz VS, Santos TL, Froufe LCM. Corredores ecológicos como ferramentas para a desfragmentação de florestas tropicais. Pesquisa Florestal Brasileira 2010; 30(63): 207-216. http://dx.doi.org/10.4336/2010.pfb.30.63.207.

Soares JCF. Uma breve história do café na região da Vila de Resende no século XIX. 2011. [citado 2011 set. 13]. Disponível em: http://www.valedoparaiba.com/terragente/ estudos/ cafe/download.pdf.

Sollberg I, Schiavetti A, Moraes MEB. Manejo agrícola no Refúgio de Vida Silvestre de Una: agroflorestas como uma perspectiva de conservação. Revista Árvore 2014; 38(2): 241-250. http://dx.doi.org/10.1590/S010067622014000200004

Stein SJ. Vassouras: um município brasileiro do café, 1850-1900. Rio de Janeiro: Editora Nova Fronteira; 1985.

Tabarelli M, Pinto LP, Silva JMC, Hirota MM, Bede LC. Desafios e oportunidades para a conservação da biodiversidade na Mata Atlântica brasileira. Megadiversidade 2005; 1(1): 132-138.

Tebaldi ALC, Fiedler NC, Juvanhol RS, Dias HM. Ações de prevenção e combate aos incêndios florestais nas unidades de conservação estaduais do Espírito Santo. Floresta e Ambiente 2013; 20(4): 538-549. http://dx.doi. org/10.4322/floram.2013.036.

Vargas AB. Diversidade de formigas em fragmentos florestais no Vale do Paraíba, Vassouras, Rio de Janeiro [tese]. Seropédica: Instituto de Florestas, Universidade Federal Rural do Rio de Janeiro; 2011.

Viana VM, Pinheiro LAFV. Conservação da biodiversidade em fragmentos florestais. Série Técnica IPEF 1998; 12(32): 25-42. 